\section{Kidney \\ Blood Pressure Research}

Review

\title{
Dialysis Exercise Team: The Way to Sustain Exercise Programs in Hemodialysis Patients
}

\author{
Alessandro Capitaninia Sara Lange ${ }^{b}$ Claudia D'Alessandro ${ }^{c}$ Emilio Salotti ${ }^{b}$ \\ Alba Tavolaro ${ }^{a}$ Maria E. Barontic Domenico Giannese ${ }^{c}$ Adamasco Cupistic

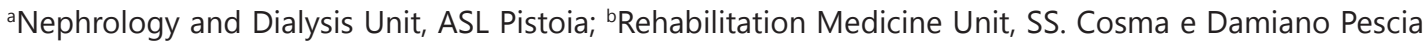 \\ Hospital, Pescia; 'Department of Clinical and Experimental Medicine, University of Pisa, Pisa, Italy
}

\section{Key Words}

Hemodialysis $•$ End-stage renal disease $\cdot$ Exercise $•$ Physical activity

\begin{abstract}
Patients affected by end-stage renal disease (ESRD) show quite lower physical activity and exercise capacity when compared to healthy individuals. In addition, a sedentary lifestyle is favoured by lack of a specific counseling on exercise implementation in the nephrology care setting. Increasing physical activity level should represent a goal for every dialysis patient care management. Three crucial elements of clinical care may contribute to sustain a hemodialysis exercise program: a) involvement of exercise professionals, b) real commitment of nephrologists and dialysis professionals, c) individual patient adaptation of the exercise program. Dialysis staff have a crucial role to encourage and assist patients during intra-dialysis exercise, but other professionals should be included in the ideal "exercise team" for dialysis patients. Evaluation of general condition, comorbidities (especially cardiovascular), nutritional status and physical exercise capacity are mandatory to propose an exercise program, in either extra-dialysis or intra-dialysis setting. To this aim, nephrologist should lead a team of specialists and professionals including cardiologist, physiotherapist, exercise physiologist, renal dietician and nurse. In this scenario, dialysis nurses play a pivotal role since they guarantee a constant and direct approach. Unfortunately dialysis staff may often lack of information and formation about exercise management while they take care patients during the dialysis session. Building an effective exercise team, promoting the culture of exercise and increasing physical activity levels lead to a more complete and modern clinical care management of ESRD patients.
\end{abstract}

Copyright $\odot 2014$ S. Karger AG, Basel

\section{Introduction}

Patients affected by end-stage renal disease (ESRD) show quite lower physical activity and exercise capacity [1-3] when compared to healthy individuals. Muscle strength 


\section{Kidney \\ Blood Pressure Research}

Kidney Blood Press Res 2014;39:129-133

DOI: 10.1159/000355787
Published onlIne: July 29, 2014

(c) 2014 S. Karger AG, Basel

www.karger.com/kbr

Capitanini et al: Exercise Team in Dialysis

and aerobic capacity are extremely limited in hemodialysis (HD) patients [4-6]. Uremic intoxication, anemia, mineral and metabolic abnormalities, cardiovascular comorbidities and uremic sarcopenia $[7,8]$ may explain the low physical performance in ESRD patients. In addition, a sedentary lifestyle and an insufficient counseling in the nephrology care setting contribute to low physical activity [9].

An increment of physical activity level should represent a goal of the clinical care management, but several barriers prevent a widespread implementation of physical exercise programs in dialysis units [10].

Three crucial elements may contribute to sustain a hemodialysis exercise program: a) involvement of exercise professionals, b) real commitment of nephrologists and dialysis professionals, c) individual patient adaptation of the exercise program.

This is in agreement with Bennet's sentence: "patients can increase the amount and the quality of exercise if the dialysis center they are attending has a culture of exercise" [11].

Despite the evidences leading to recommendations for increasing physical activity in patients with ESRD, the implementation of physical exercise programs is still uncommon in most of the dialysis units [12].

A major reason is that the HD population is quite heterogeneous from a clinical and physical point of view. Exercise programs must be tailored to physical capacity and comorbidities of each patient who must enter the decision making process. This is the main way for a correct and safe implementation of physical activity in ESRD patients [13, 14]. Dialysis nurses have a crucial role to encourage and assist patients during intra-dialysis exercise but they need to develop knowledge and skillness to assist patients during their intradialysis exercising. This strengthens the need of involving exercise professionals in an exercise team in dialysis.

In summary, evaluation of general condition, comorbidities (especially cardiovascular), nutritional status and physical exercise capacity are mandatory to propose an exercise program, during the extra-dialysis or intra-dialysis time, or both [15]. Nephrologists should lead a team of specialists and professionals including cardiologist, physiotherapist, exercise physiologists, renal dieticians and nurses. You can say.... "we have a dream"... since many dialysis center have not all these resources, but our experience allows to say.... "yes, we can"!

\section{The Exercise Team}

In our own experience, several years ago we began the implementation of physical activities in ESRD patients by an extra-dialysis program. At first, it involved only younger patients without severe comorbidities [16]. We included patients able to complete a treadmill test, performing mild to moderate training. All of the patients were candidate for receiving kidney transplantation. $\mathrm{Pa}$ tients underwent nutritional status evaluation, echocardiography and cardiopulmonary exercise test by a sports medicine physician. The training sessions were performed 2 times a week, on non-dialysis

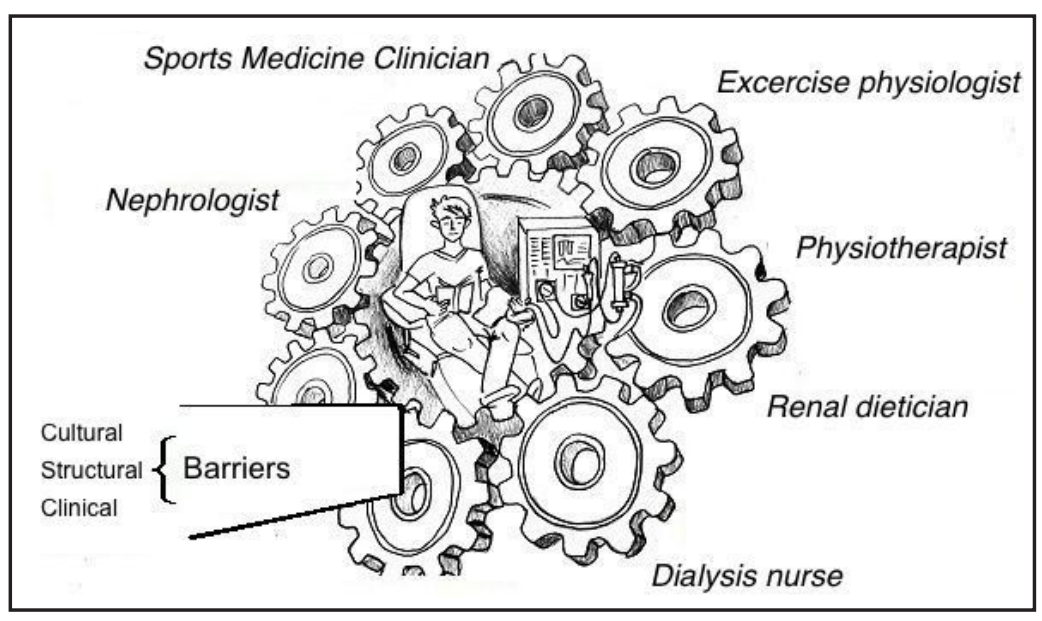

Fig. 1. The ideal Exercise team. 


\section{Kidney Blood Pressure Research}

days, for 12 months. Duration of each session was up to 90 minutes including a warm-up phase of stretching exercises (15-20 minutes) followed by 20-50 minutes of cycling on an electronically braked cycle ergometer and finally followed by a recovery phase. The intensity of the exercise was submaximal - that is, under the anaerobic threshold (S1), not exceeding $60 \%$ of peak VO2 consumption. All the sessions were held in a gym outside the hospital, with the supervision of a skilled exercise physiologist together with a dialysis nurse or a nephrologist of the dialysis unit.

The exercise training we used, namely an outpatient supervised program in a gym, was very well accepted by the patients who enjoyed this activity. No significant unwanted or side effects were recorded. A very positive aspect was the favorable psychological impact on the patients: after a few months they reported "to feel better" like "quite normal" people. However, this approach excluded elderly patients and those with severe comorbidities, who represent the majority of the common dialysis population.

Thus, with the aim to involve more patients, other types of exercise training were proposed: home exercise rehabilitation programs, exercise training in in-hospital gym or exercise activity during the course of the HD session [17].

All the patients were offered training during the HD sessions (thrice weekly). Based on assessment of the single patient capabilities, physiotherapist prepared and showed the exercise schedule, while dialysis nurses gave their support when needed. Patients performed low-intensity exercises for coordination, muscle strengthening and flexibility for $10^{\prime}$ up to 30 ' during the first 2 hours of the dialysis session. Three out of 4 patients who were unable to walk, recovered walking capacity and, 3 months later, they could perform a 6MWT.

In addition, two different programs of extra-dialysis training were carried on in stable uncomplicated HD patients, provided adaptation to the patient's clinical conditions and physical capability. Baseline physical performance was evaluated by a physiotherapist using 3 different tests: - diary and pedometer recordings of walking daily activity. - six minutes walking test (6MWT), as a well validated test of submaximal performance capacity, - treadmill test, as a measure of endurance capacity.

Then we offered, based on patient's willingness and physical capability, two types of programs:

a) advised-walking program, namely a home exercise training program

b) supervised-walking program, namely a home exercise training program plus an additional supervised training twice a week in a in-hospital gym, including a treadmill and a workout with an upper limb ergometer crank.

Patients were trained to adjust exercise intensity using the Borg scale.

In both groups, physical performance increased dramatically with time, expecially in the advised walking group. Our experience was in keeping with the data of the literature and clearly showed that physical performance can be safely enhanced in hemodialysis patients.

The engagement of experts in physical therapy (namely physiotherapist and exercise physiologist) contributed to the efficacy and safety of individually prescribed exercise programs. This can reduce this workload from the hemodialysis staff who often considered intra-dialysis exercise a disturbing factor during their duty $[18,19]$. Following nephrologist's indication and prescription, exercise professionals can coach and encourage dialysis staff to embrace exercise as a part of the patients' prescription $[20,21]$ and may contribute to increased confidence of the dialysis staff with exercising. It have been reported that if exercise professionals are not involved, dialysis staff prioritize other tasks and patient exercise rates fall down [22].

In our experience during the periods assisted by physiotherapist, the adherence to treatment programs was over $80 \%$. Unfortunately, without the presence of the therapist, focus on the activity decreased greatly in a few months.

We agree with the Authors [23] who claim that exercise professionals should be present in a dialysis unit at least for 2 days a week, so that they can see all the patients once at least. 


\section{Kidney \\ Blood Pressure Research}

Exercise professional involvement greatly contributes to increase the "exercise culture" in the hemodialysis units, the only way to create a sustaining good practice.

Another concern is the age of the patients: dialysis staff often view the HD patients as being too old to exercise. In our experience elderly hemodialysis patients responded better than younger ones to exercise programs. Younger patients often refuse adapted physical activity, feeling dwarfed by rehabilitative programs while older patients are excited to be considered and taken care. Our experience is in keeping with the statement that age is not a barrier to exercise in hemodialysis.

Finally, a renal dietician can be usefully included in the "exercise team". He or she can give individual dietary recommendations, depending on the type and duration of exercise, the distance from the dialysis session, and co-morbidities (for example diabetes). In addition renal dietician can contribute to assess nutritional status and to provide counseling focused on changes of the life style.

\section{Final Considerations}

Dialysis patients represent a quite sedentary population, and inactivity is associated with disability and poor outcomes. Unfortunately, a low frequency of physical activity assessment and exercise counseling occur in most of the dialysis units.

So the question arises on what strategies may work to enhance an effective implementation of exercise programs in our dialysis units. A first step maybe the creation of an exercise dialysis team including nephrologist, sports medicine clinician, physiotherapist, nurses, exercise physiologist and even renal dietician.

The following may be 10 suggested items for a safe and effective implementation of physical exercise in dialysis patients:

1. Involvement of exercise professionals

2. commitment by dialysis and medical staff

3. careful assessment of patient's physical capacity

4. single patient's tailored prescription of exercise program

5. implementation of intra-dialysis exercise when possible

6. avoiding repetitive or boring exercise schedules

7. exercise professionals should be present when commencing intra-dialysis exercise

8. regular assessment of patient's physical capacity

9. use of diary recall and/or pedometer to record habitual daily physical activity

10. providing of adequate equipment

A real commitment by dialysis and medical staff is mandatory. Exercise must be seen as in integral component of the clinical care management of ESRD patients. Actually, improving physical function, quality of life, psychological status other than muscle mass and strength are specific effects of exercise training. Tailoring exercise program on patients' physical capacity is essential to prevent feelings of inadequacy and for a correct and safe implementation of physical activity.

\section{Disclosure Statement}

The authors have no sponsorship or funding arrangements relating to their research, nor conflicts of interest. 


\section{Kidney \\ Blood Pressure Research}

\begin{tabular}{l|l}
\hline Kidney Blood Press Res 2014;39:129-133 \\
\hline DOI: 10.1159/000355787 & $\begin{array}{l}\text { C) 2014 S. Karger AG, Basel } \\
\text { www.karger.com/kbr }\end{array}$ \\
\hline Published online: July 29, 2014 &
\end{tabular}

\section{References}

1 Heiwe S, Jacobson SH: Exercise training for adults with chronic kidney disease. Cochrane Database Syst Rev 2011;10:CD003236.

2 Painter P, Roshanvaran B: The association of physical activity and physical function with clinical outcomes in adults with chronic kidney disease. Curr Op Nephrol Hypert 2013;22:615-623.

-3 Johansen KL, Kaysen GA, Dalrymple LS, Grimes BA, Glidden DV, Anand S, Chertow GM: Association of Physical Activity with Survival among Ambulatory Patients on Dialysis: The Comprehensive Dialysis Study. Clin J Am Soc Nephrol 2013;8:248-253.

-4 Roshanravan B, Robinson-Cohen C, Patel KV, Ayers E, Littman AJ, de Boer IH, Ikizler TA, Himmelfarb J, Katzel LI, Kestenbaum B, Seliger S: Association between physical performance and all-cause mortality in CKD. J am Soc Nephrol 2013;24:822-830.

-5 Painter P, Messer-Rebak D, Hanson P: Exercise capacity in hemodialysis, CAPD and renal transplant patients. Nephron 1986;42:47-51.

6 Johansen KL, Chertow GM, Ng AV, Mulligan K, Carey S, Schoenfeld PY, Kent-Braun JA: Physical activity levels in patients on hemodialysis and healthy sedentary controls. Kidney Int 2000;57:2564-2570.

7 Moore G, Parsons D, Stray-Gundersen J, Painter P, Brinker K, Mitchell J: Uraemic myopathy limits aerobic capacity in haemodialysis patients. Am J Kidney Dis 1993;22:277-287.

8 Fahal IH: Uraemic sarcopenia: aetiology and implications. Nephrol Dial Transplant 2013, DOI 10.1093/ ndt/gft070.

-9 Delgado C, Johansen KL: Deficient counseling on physical activity among nephrologists. Nephron Clin Pract 2010;116:330-336.

10 Stack A, Murthy B: Exercise and limitations in physical activity levels among new dialysis patients in the United States: an epidemiologic study. Ann Epidemiol 2008;18:880-888.

-11 Bennet P: How a culture of exercise benefits patients on dialysis. J Renal Nursing 2010;2:64-68.

12 Manfredini F, Mallamaci F, Catizoni L, Zoccali C: The burden of physical activity in chronic kidney disease: is there an exit strategy? Nephrol Dial Transplant 2012;27:2143-2145.

13 Delgado C, Johansen KL: Barriers to exercise participation among dialysis patients. Nephrol Dial Transpl 2012;27:1152-1157.

14 Johansen KL: Exercise and dialysis. Hemodial Int 2008;12:290-300.

15 Cupisti A, Capitanini A, Betti G, D’Alessandro C, Barsotti G: Assessment of habitual physical activity and Energy expenditure in dialysis patients and relationships to nutritional parameters, Clin Nephrol 2011;75:218-225.

-16 Capitanini A, Cupisti A, Mochi N, Rossini D, Lupi A, Michelotti G, Rossi A: Effects of exercise training on exercise aerobic capacity and quality of life in hemodialysis patients. J Nephrol 2008;21:738-743.

17 Bulckaen M, Capitanini A, Lange S, Caciula A, Giuntoli F, Cupisti A: Implementation of exercise training programs in a hemodialysis unit: effects on physical performance. J Nephrol 2011;24:790-797.

18 Carlson L, Carey S: Staff responsibility to exercise. Adv Renal Replace Ther 1999;6:172-180.

19 Forgeron S, Valeriote L: Keeping our patients pedaling - one unit's experience. CANNT J 2001;11:19-21.

-20 Cheema B, Abas H, Smith B, O’Sullivan A, Chan M, Patwardhan A, Kelly J, Gillin A, Pang G, Lloyd B, Singh MF: Progressive exercise for anabolism in kidney disease (PEAK): a randomized, controlled trial of resistance training during Hemodialysis. J Am Soc Nephrol 2007;18:1594-1601.

21 Johansen K: Exercise in the end-stage renal disease population. J Am Soc Nephrol 2007;18:1845-1854.

-22 Bennett PN, Breugelmans L, Barnard R, Agius M, Chan D, Fraser D, McNeill L, Potter L: Sustaining a hemodialysis exercise program: a review. Semin Dial 2010;23:62-73.

23 Painter P, Carlson L, Carey S, Myll J, Paul S: Determinants of exercise encouragement practices in hemodialysis staff. Nephrol Nurs J 2004;31:67-74. 